\title{
WELTLICHE EINFLÜSSE IN DER NOTRE-DAME-MUSIK? ÜBERLEGUNGEN ZU EINER KLAUSEL IM CODEX F ${ }^{1}$
}

Fred BÜTTNER

\begin{abstract}
The unlimited validity of the rectilinear evolution that, according to Friedrich Ludwig, led from Notre Dame Clausula to Latin and thenceforth to French Motet was most recently questioned by Wolf Frobenius in his article "Zum genetischen Verhältnis zwischen Notre-Dame-Klauseln und ihren Motetten" (1987). Nevertheless, Frobenius does not thoroughly examine the pieces concerned, with hardly more far-reaching conclusions being attached to the priority claimed for many French Motets when comparing their concordances as Clausula and Latin Motet. With a setting of the choral melisma "ne" (Sm8) - already used by Ludwig in order to substantiate his estimation of the historical precedence of the Clausula - as an example, the point of view held by Frobenius is therefore once again subjected to critical scrutiny. Thus several traits can be established in the specifics of the Clausula that point to the field of secular music. Although this corroborates the considerations made by Frobenius, on the other hand such a result will open up new perspectives for further research into the School of Notre Dame.
\end{abstract}

\section{Zusammenfassung}

Spätestens durch den Aufsatz „Zum genetischen Verhältnis zwischen Notre-Dame-Klauseln und ihren Motetten“ von Wolf Frobenius (1987) wurde die uneingeschränkte Gültigkeit des geradlinigen Entwicklungsgangs, den Friedrich Ludwig von der Notre-Dame-Klausel über die lateinische bis zur französischen Motette zeichnete, in Frage gestellt. Jedoch bleibt Frobenius eine gründliche Untersuchung der betreffenden Stücke ebenso schuldig, wie auch an die behauptete Priorität vieler französischer Motetten gegenüber ihren Konkordanzen als Klausel und lateinische Motette kaum weiterreichende Schlußfolgerungen geknüpft werden. Anhand des „Ne“-Satzes Sm8, den bereits Ludwig exemplarisch hervorhob, um seine Auffassung von der Klausel als ältestem Stadium zu belegen, wird deshalb die Sichtweise von Frobenius noch einmal auf ihre Stichhaltigkeit überprüft. Dabei lassen sich im Klauselsatz verschiedene Züge festmachen, die auf den Bereich der weltlichen Musik deuten. Gibt dieses Ergebnis einerseits Frobenius Recht, so eröffnet es andererseits Perspektiven, aus denen neue Aufgaben für die Notre-Dame-Forschung abgeleitet werden können.

Als Friedrich Ludwig, der große Ahnherr der Mediävistik im Fach Musikwissenschaft, vor etwa hundert Jahren daranging, die Mehrstimmigkeit des späten 12. und des 13. Jahrhunderts zu erschließen, sah er sich, neben der rein philologischen Erfassung der vorhandenen Quellen

1. Vortrag, gehalten am 19. Juli 2000 an der Friedrich-Schiller-Universität Jena. - Beim Codex F, einer im 13. Jahrhundert in Paris entstandenen und bereits im späten Mittelalter nach Florenz gelangten Handschrift (heute I-Fl, Plut.29,1), handelt es sich um die zentrale Quelle für das Schaffen der sog. ,Notre-Dame-Schule'. Eine von Luther Dittmer herausgegebene Faksimile-Ausgabe in zwei Teilen (Firenze, Biblioteca mediceo-laurenziana, Pluteo 29, I: Teil I. Faszikel IVI und Teil II. Faszikel VII-XI) liegt als Band 10-11 im Rahmen der Reihe Veröffentlichungen mittelalterlicher Musikhandschriften vor.

Anuario Musical, 57 (2002) 
und ihres Inhalts, auch vor die Aufgabe gestellt, den damals noch weitgehend im Dunkeln liegenden Entwicklungsgang der mittelalterlichen Musikgeschichte im genannten Zeitraum grundsätzlich, d. h. in seinen Hauptzügen, offen zu legen. Zu diesem Zweck beschrieb er als wesentliche Stationen des musikalischen Fortschritts das zweistimmige Organum der NotreDame-Schule von Paris, das Austauschen der ursprünglichen Discantuspartien im Organum durch neuere Ersatzklauseln, deren Tropierung mit einem geistlichen Text auf Lateinisch im Duplum (der zum gregorianischen Choral im Tenor hinzutretenden Stimme) und schließlich ihre Loslösung aus dem Organum, die mit einer späteren, nun jedoch weltlichen Textierung auf Französisch einhergeht. ${ }^{2}$

Die Stringenz dieses Übergangs von der alten Organalkunst in ihrer reifsten Ausprägung zur frühen ,Motette ' mit textiertem Duplum hat ohne Zweifel etwas Bestechendes, und sie steht, aufs Ganze gesehen, durchaus im Einklang mit der Quellenlage. In der Folgezeit aber konnte namentlich William G. Waites Untersuchung The Rhythm of Twelfth-Century Polyphony auf mehrere Klauseln hinweisen, die ihrerseits den Eindruck vermitteln, als habe man sie durch Enttextierung aus einer vorhandenen Motette - in der überwiegenden Zahl der Fälle offenbar einer weltlichen Motette mit französischem Text - gewonnen. ${ }^{3}$ Das von Ludwig noch sauber getrennte Stadium der geistlichen Notre-Dame-Musik und des weltlichen Motettenschaffens begaben sich so in wechselseitigen Austausch, und das eindimensionale Nacheinander historischer Epochen verwandelte sich zu fruchtbarem Miteinander.

Welche Konsequenzen haben wir jedoch aus der Differenzierung im Verhältnis von Klausel und Motette zu ziehen? Ich denke, es ist einsichtig, dass die Möglichkeit von weltlichen Motettenvorlagen für Notre-Dame-Klauseln in erster Linie eine neue Voraussetzung für unsere Beschäftigung mit der Notre-Dame-Musik selbst schafft, denn während seit Ludwig eine Abhängigkeit der weltlichen Motette von der Notre-Dame-Klausel außer Frage stand (wie anders sollte man auch das Vorhandensein eines gregorianischen Melismas im Tenor von weltlichen Motetten erklären?), haben wir jetzt, zumindest ab einem gewissen Zeitpunkt, in entsprechender Weise mit weltlichen Einflüssen auf die Notre-Dame-Musik zu rechnen. Das aber scheint mir

2. Siehe etwa Ludwigs Aufsatz „Studien über die Geschichte der mehrstimmigen Musik im Mittelalter. III. Über die Entstehung und die erste Entwicklung der lateinischen und französischen Motette in musikalischer Beziehung", in: SIMG 7 (1905-1906), S. 514-528 und die „Einleitung“ zum Repertorium organorum recentioris et motetorum vetustissimi stili, Bd. I/1, Halle 1910, S. 1-6.

3. William G. Waite, The Rhythm of Twelfth-Century Polyphony. Its Theory and Practice (= Yale Studies in the History of Music 2), New Haven 1954, S. 100 f. - Schon Jahre vorher ging Heinrich Husmann in seiner kritischen Gesamtausgabe der drei- und vierstimmigen Notre-Dame-Organa (= Publikationen älterer Musik 11), Leipzig 1940, S. 133 davon aus, dass der Notator einer dreistimmigen „Tanquam“-Klausel in F (f. 10v) als Vorlage neben einer Klauselversion auch eine Motettenaufzeichnung benützt hat, und Klaus Hofmann, Untersuchungen zur Kompositionstechnik der Motette im 13. Jahrhundert (= Tübinger Beiträge zur Musikwissenschaft 2), Neuhausen-Stuttgart 1972, S. 129 konstatiert, wenn auch nur indirekt, die Abhängigkeit einer zweistimmigen „In seculum“-Klausel in F (f. $157^{\circ}$ ) von ihrer konkordanten Motette „Lonc tens ai mon cuer assis/In seculum“, indem er die Disposition ihres Tenors als ,refrainbedingt" bewertet, wie er angesichts der „,satztechnischen Bedeutung der Motettenrefrains“ allgemein an künftige Forschungen appelliert, ,das Problem der Priorität von Klausel oder Motette neu zu überprüfen“ (ebd., S. 122, Anm. 23). 
eine aufregende Perspektive zu sein, deren Tragweite von der heutigen Musikwissenschaft, wenn überhaupt, so nur in Ansätzen wahrgenommen wird. ${ }^{4}$

In diesem Sinne geht auch der bislang mutigste Vorstoß, um die Priorität von französischen Motetten gegenüber ihren Klauselkonkordanzen im 13. Jahrhundert festzumachen, der 1987 erschienene Aufsatz „Zum genetischen Verhältnis zwischen Notre-Dame-Klauseln und ihren Motetten“ von Wolf Frobenius, ${ }^{5}$ über eine Aufzählung aller relevant scheinenden Stücke mit lediglich kurzem Hinweis auf das Argument, welches für die Motettenpriorität spricht, kaum hinaus. Dabei werden die Notre-Dame-Klauseln mit denjenigen der Handschrift Saint-Victor ${ }^{6}$ bunt zusammengewürfelt, obschon zu diesem Zeitpunkt noch völlig unklar war, in welcher Beziehung denn das Klauselkorpus von Saint-Victor zur Notre-Dame-Schule überhaupt steht. Inzwischen konnte ich selbst im Rahmen meiner Habilitationsschrift wahrscheinlich machen, dass die vierzig Klauseln der Handschrift Saint-Victor erst im späteren 13. Jahrhundert durch Enttextierung französischer Motetten entstanden, wobei sie einem konservativen Kreis von Musikern dazu dienten, den mehrstimmigen Satz exemplarisch aus dem weltlichen Ambiente in die Kirche zurückzuholen, von wo er seinen Ausgang genommen hatte. ${ }^{7}$ Dass jedoch die NotreDame-Musiker bei Verwendung französischer Motetten als Klauselvorlage dieselben Intentionen

4. Ausgehend von der ca. 1210 verfassten Summa des Robert de Courson hat Christopher Page in seinem Buch The Owl and the Nightingale. Musical Life and Ideas in France 1100-1300, London 1989, S. 144-154 interessante Überlegungen zur Nachbarschaft von geistlicher und weltlicher Musik in der Tätigkeit der damaligen „,magistri organici“ angestellt, wobei Coursons hartes Urteil über den „,prelatus lascivus“, welcher Angehörigen dieses Musikerstandes erlaube, dass sie „,scurrilia et lascivia [...] in ecclesia sua" zu Gehör bringen, und die Weisung, dass ihre Organalkunst im Gottesdienst nur toleriert werden solle, „dummodo scurriles notule non admisceantur", ebenfalls das Vorhandensein weltlicher Einflüsse in der mehrstimmigen Kirchenmusik des beginnenden 13. Jahrhunderts unterstreichen. Page bezieht die Textstellen dann jedoch nur auf ,improvised embellishments which [...] were used by performers of the two-part organum“ (ebd., S. 147), während er andererseits Coursons Ansicht, ,quod illicite sunt opere magistrorum organicorum qui scurrilia et effeminata proponunt iuvenibus et rudibus ad effeminandos animos ipsorum", zum Anlass nimmt, um weiterhin die französische Motette der frühesten Zeit ausschließlich mit einer Neutextierung von Klauseln und der damit verbundenen Abwanderung dieser Musik in den weltlichen Bereich zu erklären: „What could be more natural than that singers who were trying to make a living from their voices and from their polyphonic expertise should have seen an opportunity to locare operas suas in a secular context by the simple device of adding vernacular words to clausulae, performing the results before a secular audience - one which a moralist like Courson was all too ready to dismiss as a collection of ,young and ignorant persons"?" (Ebd., S. 154) Dass französische Motetten, ihres Textes entkleidet, in das Klauselrepertoire übernommen worden sein könnten, findet dabei keine Berücksichtigung.

5. Wolf Frobenius, ,Zum genetischen Verhältnis zwischen Notre-Dame-Klauseln und ihren Motetten“, in: $A f M w 44$ (1987), S. 1-39. Nach Untersuchung der „Klauseln mit französischen Motetten“ wendet sich der Aufsatz zwar auch den „Klauseln mit ausschließlich lateinischen Motetten“ zu, jedoch lässt sich bereits an diesen Kategorien ablesen, dass Frobenius der Möglichkeit von französischen Motettenvorlagen den Vorrang gibt.

6. Unter der Bezeichnung Saint-Victor $=$ St.V versteht man eine Sammelhandschrift, die im 14. Jahrhundert von Jacques Bauchant, einem hoch gestellten Beamten (,sergant darmez“) des französischen Königs Charles V., aus zehn Einzelteilen zusammengesetzt wurde. Später gelangte sie in die Bibliothek der Pariser Abtei Saint-Victor, wo man bereits im 16. Jahrhundert ihren Anfang (f. 1-175) entnahm. Die zweite Hälfte der Handschrift (f. 176-305), die auch den Musikfaszikel enthält, wird heute in der Pariser Bibliothèque nationale aufbewahrt (F-Pn, lat.15139). Schwarzweißphotos der vierzig im Musikfaszikel überlieferten Klauseln wurden von Friedrich Gennrich, Sankt Viktor Clausulae und ihre Motetten (= Musikwissenschaftliche Studienbibliothek 5 und 6), Darmstadt 1953, und Ethel Thurston, The Music in the St. Victor Manuscript Paris lat. 15139. Polyphony of the Thirteenth Century (= Pontifical Institute of Mediaeval Studies. Studies and Texts 5), Toronto 1959, veröffentlicht. Ein Farbfaksimile bietet der in Anm. 7 genannte, von mir herausgegebene Band Die Klauseln der Handschrift Saint-Victor.

7. Fred Büttner, „Studien zum Klauselrepertoire der Handschrift Saint-Victor (Paris, BN, lat. 15139)“ (maschr. Habilitationsschrift; München 1998). Die Darstellung basiert auf den Ergebnissen eines von mir durchgeführten Arbeitsprojekts am Musikwissenschaftlichen Institut der Universität München, dessen Schlussbericht inzwischen erschienen ist (Die Klauseln der Handschrift Saint-Victor [Paris, BN, lat. 15139], hrsg. von Fred Büttner, Tutzing 1999). 
verfolgten, wäre zuerst einmal nachzuweisen. Untersucht man das Notre-Dame-Repertoire genauer, so drängt sich vielmehr der Eindruck auf, dass das Verhältnis der Notre-Dame-Musiker zum weltlichen Bereich von einer großen Offenheit geprägt war, die ebenso, wie Errungenschaften der Notre-Dame-Musik in den weltlichen Bereich hineinwirken konnten, auch das Antlitz der Notre-Dame-Musik verwandelte.

Dies ist jedoch die Kernfrage, die angesichts einer Übernahme französischer Motetten als Klausel in das Notre-Dame-Repertoire gestellt werden muss: Welchen Gewinn durften die NotreDame-Musiker von der Einbeziehung weltlicher Vorlagen für ihre eigene Sakralkunst erhoffen, und welche Impulse strahlten damit aus dem weltlichen in den geistlichen Bereich aus? Solche Ansätze wurden bisher von der Forschung nicht verfolgt, und auch die knappen Hinweise von Frobenius, um die Rückführung einzelner Klauseln auf französische Motetten zu begründen, bieten dafür oftmals keine Grundlage - ja, sie reichen bei näherem Hinsehen nicht einmal aus, um die Priorität der Motette zweifelsfrei abzusichern. In vielen Fällen begnügt sich Frobenius damit, den Einbau eines Refrains in den musikalischen Satz festzuhalten, ohne jemals weitere Fragen nach dessen genauer Gestalt in der Klausel, ihrer konkordanten Motette und gegebenenfalls seiner sonstigen Überlieferung zu stellen. Doch wäre letztlich nur auf diesem Weg zu beweisen, ob ein Refrain als präexistentes Gebilde in den Satz integriert wurde oder, wie von Ludwig stets behauptet, zuerst als bloß musikalische Schlusswendung des Duplum in der Klausel existierte und von dort, nachträglich mit Text versehen, Eingang in die Produktion der weltlichen Motette und dann vielleicht auch der weltlichen Liedkunst fand.

Auf ähnlich schwachen Füßen steht die Argumentation von Frobenius häufig mit ihren zusätzlich beigebrachten Indizien. So heißt es beispielsweise zum zweiten, nach Norman E. Smith als Sm8 bezeichneten „Ne“-Satz im großen Klauselfaszikel der Handschrift F und der mit ihm konkordanten Motette „En mai qant nest la rosee/Ne“, nach Ludwigs Repertorium ${ }^{9}$ die Nummer [62]: „In [62] ist der Schluß der zweiten Tenordurchführung zum Zitat des sehr verbreiteten Refrains, der mit gleicher Melodie auch in den Motetten [188], [403] und [433] sowie in der Pastourelle R 2041 von Pierre de Corbie vorkommt, gedehnt. Dies schließt aus, was an sich schon höchst unwahrscheinlich ist, nämlich daß - wie Ludwig, Gennrich und Kuhlmann ohne weiteres annehmen - die Klausel Sm8 die musikalische Quelle der Motette [62] und des Refrains ist." ${ }^{\text {"10 }}$ Nehmen wir, anders als Frobenius, den rhythmisch gedehnten Schluss des Tenors (genau handelt es sich um ein Abbremsen des 1. Modus zu perfekten Longen für die drei letzten Töne) vorurteilslos zur Kenntnis, so will uns jedoch keineswegs einleuchten, warum die Verlangsamung eines musikalischen Vorgangs am Ende per se über die Priorität der einen oder anderen Fassung entscheiden soll, kennen wir das Phänomen des Schlussritardando (sei es als reine Aufführungsgepflogenheit oder bereits in der Komposition realisiert) doch ebenso aus der späteren

8. Norman E. Smith, „From Clausula to Motet: Material for Further Studies in the Origin and Early History of the Motet", in: MD 39 (1980), S. 38.

S. 11 .

9. Ludwig, Repertorium organorum recentioris et motetorum vetustissimi stili, Bd. Il, Hildesheim/New York 1972,

10. Frobenius, „Zum genetischen Verhältnis zwischen Notre-Dame-Klauseln und ihren Motetten“, S. 14. 
Musikgeschichte, ohne dass wir auf den Gedanken kämen, daraus das „genetische Verhältnis“ unterschiedlicher Fassungen eines Stücks (wenn solche überhaupt vorliegen) abzuleiten.

Nachdem Ludwig für seine Betrachtungen über die ,älteste lateinische und französische Motette“ in Adlers Handbuch der Musikgeschichte gerade diesen „Ne“-Satz aus F und seine Konkordanzen (neben der französischen gibt es zwei lateinische Motetten) heranzog, um zu demonstrieren, wie durch Textierung einer Notre-Dame-Klausel ein Refrain entsteht, der sich über das französische Motettenrepertoire bis in weltliche Lieder fortpflanzen kann, ${ }^{11}$ scheint es lohnend, das Beispiel des „Ne“-Satzes nochmals auf den Prüfstand zu stellen und dabei nach hieb- und stichfesten Argumenten zu suchen, mit denen die Priorität der Klausel oder einer Motettenversion nachgewiesen werden kann. Sollte sich die französische Motette tatsächlich als Ausgangspunkt der übrigen Fassungen erweisen, so müssten ebenjene Merkmale, die den Vorrang der französischen Motette untermauern, aber auch auf ihre besondere, sich von der Faktur einer originären Klausel abhebende Qualität befragt werden, um damit das weltliche Moment im Repertoire der Notre-Dame-Schule festzumachen und die Veränderungen, die sich für diese Musik aus dem weltlichen Einfluss ergeben, in ihrer Bedeutung abschätzen zu können.

Beispiel 1:

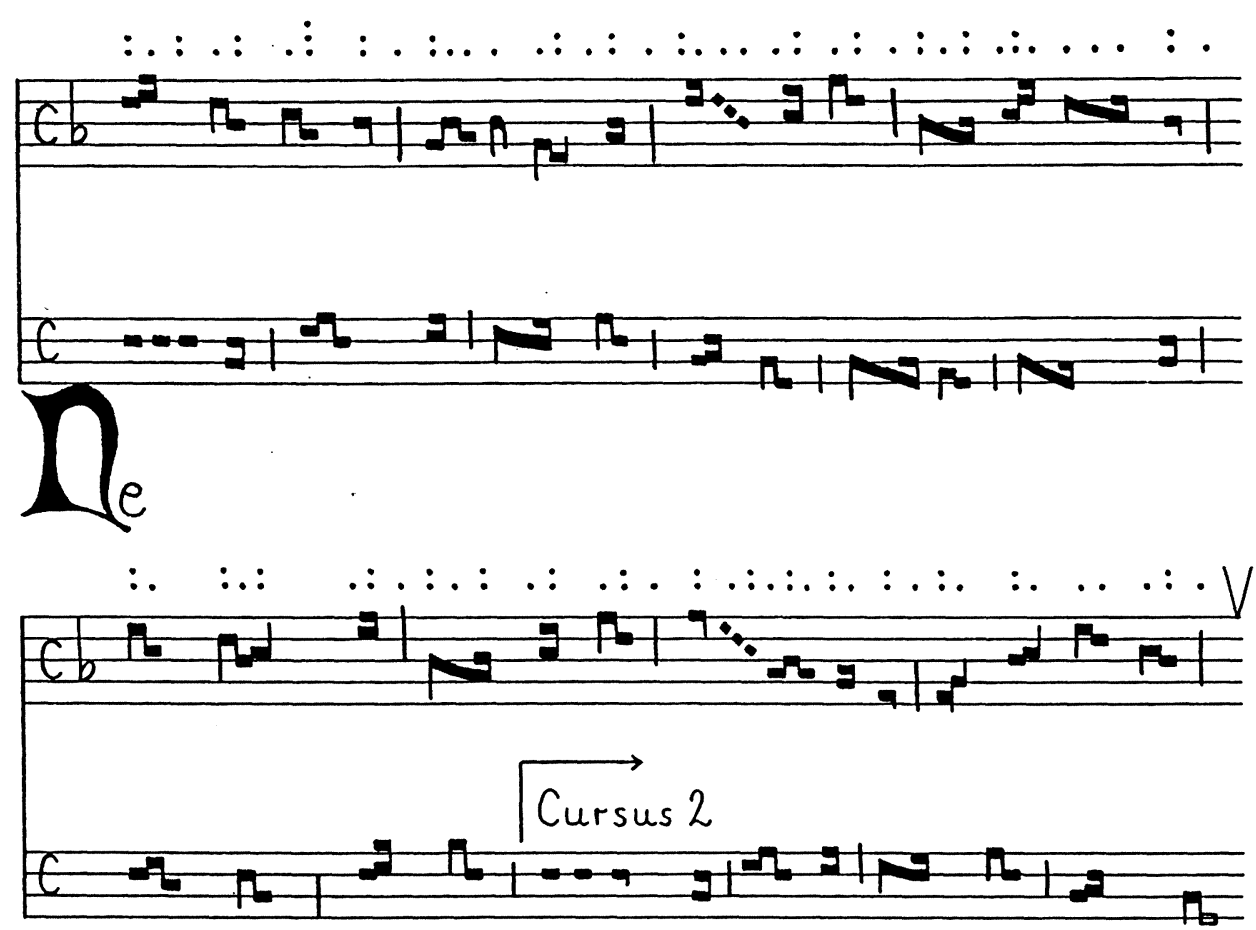

11. Ludwig, „Die geistliche nichtliturgische und weltliche einstimmige und die mehrstimmige Musik des Mittelalters bis zum Anfang des 15. Jahrhunderts“", in: Handbuch der Musikgeschichte, hrsg. von Guido Adler, Frankfurt am Main 1924, S. 205-207. 


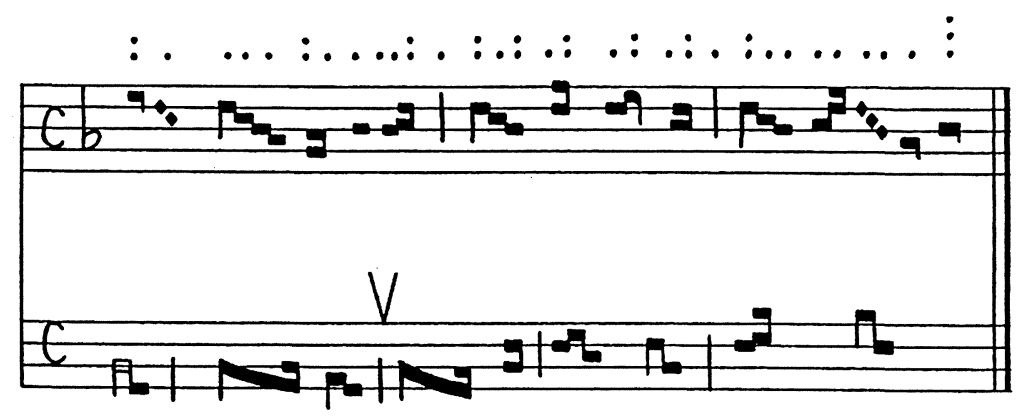

Nachschrift der Klausel Sm8
aus der Handschrift F, f. $151^{\text {r }}$

Wie alle weiteren Nachschriften, so behält auch Beispiel 1 die Notenzeichen der Quelle bei; lediglich der vertikale Bezug der Stimmen wurde, wo es nötig schien, verbessert. Die Zeilenumbrüche sind in Übereinstimmung mit der formalen Gliederung des Stücks gesetzt, jedoch wird der originale Zeilenumbruch jeweils mit einem $\vee$ über dem Notensystem angezeigt (der Seitenumbruch in Beispiel 4 mit einem doppelten $\vee$ ); Ligaturen, die den Zeilenumbruch mitvollziehen, erscheinen am Ende des ersten und am Anfang des zweiten Notensystems, wobei nicht erklingende Töne hohl bleiben. Die Punkte geben den angenommenen Wert der einzelnen Töne in Brevisdauern an, d. h. ein Punkt bedeutet eine Brevis recta, zwei Punkte eine Brevis altera oder Longa imperfecta und drei Punkte eine Longa perfecta; in der Notation durch Striche mitgeteilte Plicae werden dem ausgeschriebenen Ton zugerechnet, zu dem sie gehören.

Fassen wir zuerst die Phase des musikalischen Vorgangs ins Auge, die in der Diskussion um Klausel- oder Motettenpriorität von jeher die größte Rolle gespielt hat: den Schluss mit seiner Refrainmelodie. Tatsächlich treten hier musikalische Bauprinzipien in Erscheinung, die für einen Großteil der im 13. Jahrhundert begegnenden Refrains typisch sind - das Gliedern in zwei Hälften (in der Klauselnotierung die beiden letzten Ordines), denen auf der Textebene zwei Verse zugeordnet werden, und der gleiche Anlauf der Hälften mit unterschiedlichem Ende. Im vorliegenden Fall beginnen die Hälften jedes Mal mit $e^{\prime}-d^{\prime}-c^{\prime}$, während die erste Hälfte offen auf dem Anfangston $e$ ' schließt, nach dem ja auch das anfängliche $e^{\prime}-d^{\prime}-c^{\prime}$ zu Beginn der zweiten Hälfte wiederkehrt, und die zweite Hälfte zu einem vollgültigen Schluss auf dem unteren Ton des $e^{\prime}-d^{\prime}-c^{\prime}$-Abstiegs, also $c^{\prime}$, findet.

Diese für einen Refrain des 13. Jahrhunderts typische Melodie tritt nun also, worauf schon Ludwig in seinem Beitrag aus dem Handbuch der Musikgeschichte hinweist, mit einem ebenso typischen Refraintext, ,,cele ma samor donee/qi mon cuer et mon cors a“, nicht nur in der französischen Motettenkonkordanz zu unserer „Ne“-Klausel sowie drei anderen Motetten auf (davon einmal in der dritten Stimme, dem Triplum), sondern auch einem weltlichen Lied des Trouvère Pierre de Corbie. Da Refrains normalerweise jedoch ihre Refrainfunktion, $d$. h. die unveränderte Wiederkehr nach einem festgelegten Formschema, allein im Kontext der weltlichen Liedkunst ausüben, wohingegen sich ihr Einsatz in der Motette zumeist auf vereinzeltes 
Einblenden beschränkt (häufig am Schluss, der auch in weltlichen Liedern, ungeachtet aller formalen Unterschiede der Gattungen, den Refrain nochmals bringt), sollten wir annehmen, dass unser Refrain am ehesten aus dem Lied des Pierre de Corbie übernommen ist. Eine solche Annahme wird aber durch den Umstand entkräftet, dass dieses Lied zu keiner der fraglichen Refraingattungen Rondeau, Virelai und Ballade gehört, sondern einen fiktiven Dialog zwischen dem Trouvère selbst und der Figur Robins realisiert, dessen Strophen - nicht anders als Motettentexte - zuletzt in das jeweils vereinzelte Einblenden eines Refrains auslaufen. ${ }^{12}$

Beispiel 2:

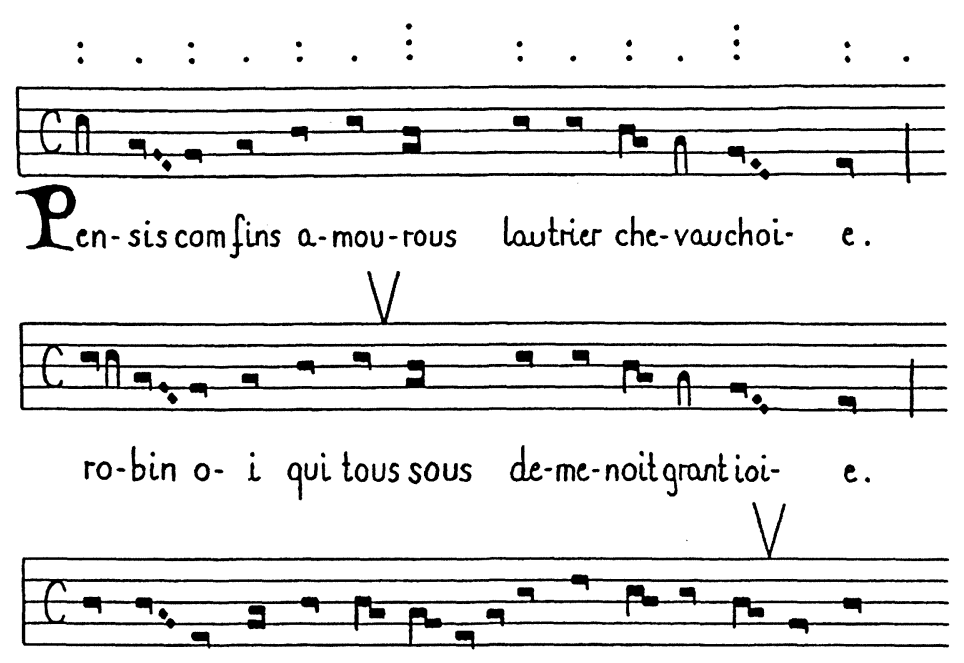

ce-le part vingsel sal-va-i.etdel re-vel li de-mandai

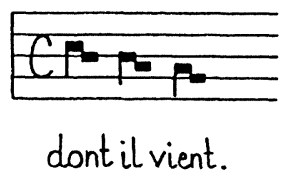

12. Das in G. Raynauds Bibliographie des altfranzösischen Liedes, Bd. 1, hrsg. von Hans Spanke (= Musicologica 1), Leiden 1980, S. 274 als Nr. 2041 geführte Lied ist zweimal überliefert, im Chansonnier Noailles (F-Pn, fr.12615 [N], f. $122^{r}-122^{v}$ ) und im Chansonnier du Roi (F-Pn, fr.844 [R], f. $20^{r}-21^{r}$ ). Wie für Strophenlieder allgemein üblich, versehen beide Aufzeichnungen nur die erste Strophe mit musikalischer Notation, während von den nachfolgenden Strophen lediglich der Text mitgeteilt wird; einzige Ausnahme bilden die wechselnden Refrains jeweils am Strophenschluss, die melodisch voneinander abweichen. Die Nachschrift der ersten Strophe in Beispiel 2 beruht auf der Version im Chansonnier Noailles, die einen durchgehenden $g$-Modus ausprägt und damit tonal schlüssig ist. Im Vergleich hierzu beginnt die Aufzeichnung im Chansonnier du Roi einen Ton tiefer, d. h. auf $f$, das mit der absteigenden Quart $b$ - $f$ in der Kurzzeile „dont il vient" nochmals bestätigt wird (die auf $g$ stehende Version des Chansonnier Noailles schließt demgegenüber an dieser Stelle mit dem offenen a). Erst danach, wenn sich die Melodie in ihrem Schlussteil dem Refrain nähert, bringt auch der Schreiber des Chansonnier du Roi seine Notierung auf $g$-Niveau, was jedoch allem Anschein nach gewisse Unsicherheiten bei ihm auslöste, da er für den Übergang zum Refrain (,,-son i a/Cele ma samour donee“) zusätzliche Notenlinien zog und dem Refrainbeginn, obwohl dieser nicht am Anfang einer Zeile steht, einen c3-Schlüssel vorausgehen lässt. 


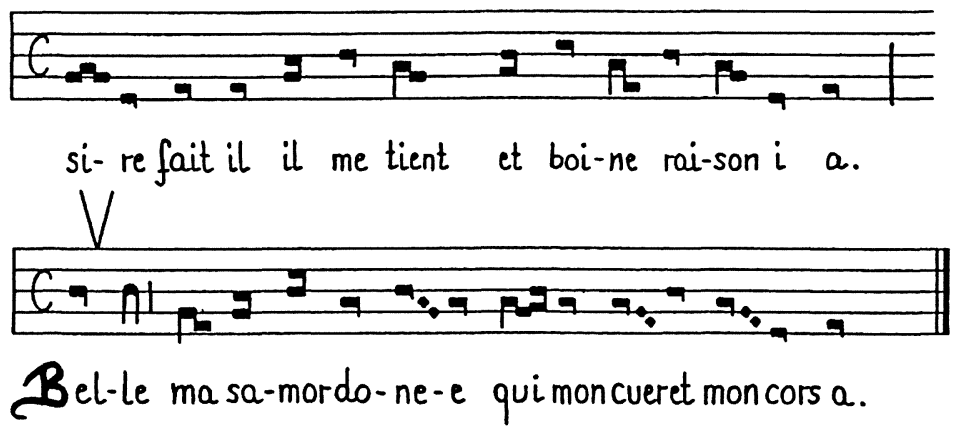

Nachschrift des Lieds R 2041 aus der Handschrift N, f. $122^{r}$

Suchen wir hingegen nach einem Stück, das den Refrain in seiner Refrainfunktion zeigt, so wird unsere Aufmerksamkeit in diesem Fall überraschend in den Bereich der französischen Motette zurückgelenkt: So prägt der im Chansonnier Noailles enthaltene Motetus „Cest la ius en la roi pree“ unter Einsatz des Refrains „cele ma samor donee/qi mon cuer et mon cors a“ ein vollständiges Rondeau aus, ${ }^{13}$ was erwartungsgemäß auf Kosten des Tenors geht, der nicht mehr - wie sonst üblich - in der Lage ist, das gregorianische Melisma in einem festen Rhythmusmodell regulär durchzuführen, und anscheinend den Ablauf der Tonfolge auch formal dem Rondeau anpasst. ${ }^{14} \mathrm{Ob}$ die Motette damit als Ursprungsort des Refrains betrachtet werden darf, ist natürlich nicht ohne weiteres zu entscheiden, doch wird sie von allen Stücken, die den Refrain

13. Eine englische Übersetzung des „Cest la ius“"-Textes bietet Mark Everist, „The Rondeau Motet: Paris and Artois in the Thirteenth Century“, in: ML 69 (1988), S. 13 bzw. French Motets in the Thirteenth Century. Music, Poetry and Genre (= Cambridge Studies in Medieval and Renaissance Music 3), Cambridge 1994, S. 100. Fälschlicherweise ist das Wort „,cors“ im Refrain hier jedoch mit „,soul“ (anstatt „,body“) wiedergegeben.

14. Ähnlich den meisten übrigen Motetten des Chansonnier Noailles und Chansonnier du Roi liefert auch im Falle von „Cest la ius en la roi pree/Pro patribus“ (N, f. 195') die Notierung des Tenors in dreitönigen Ordines (nur im vorletzten Ordo reduziert sich die Zahl der Töne auf zwei, $d^{\prime}$ und $c^{\prime}$ ) keine ausreichenden Anhaltspunkte für den gemeinten Rhythmus, da sich das „Pro patribus“-Melisma aus dem Graduale „Constitues eos“ weder in Dreitongruppen noch in einem anderen der gängigen Rhythmusmodelle sinnvoll mit dem Rondeau verbinden lässt. Die für Beispiel 3 gewählte Lösung geht von der Annahme aus, dass sich Rhythmus und Gliederung des Tenors, wie in der berühmten Motette „Mout me fu grief/Robin maime/Portare", dessen Motetus ein weitgehend mit der achtzeiligen Rondeauform übereinstimmendes Lied enthält, dem Motetusablauf flexibel anpassen. Dabei kommt es zu einer Disposition, welche die drei Phasen des „Pro patribus"-Melismas geschickt zu den Stationen des Rondeau in Beziehung setzt: Zuerst werden die beiden separaten Verszeilen a und A jeweils mit Phase 1 des Melismas versorgt, anschließend die zweimalige Kombination ab bzw. AB jeweils mit Phase 2-3. Dass die Verse a und A einmal von Phase 1, und das andere Mal von Phase 2 begleitet werden können, hängt damit zusammen, dass die drei Phasen eine latente aa'b-Form ausbilden, wie ich schon in meinem Beitrag über „Klausel Nr. 23“ in Die Klauseln der Handschrift Saint-Victor (Paris, BN, lat. 15139), S. 178 bemerkte. Obwohl also die Tenorgestalt für die Verse a und A in der ersten und zweiten Hälfte des Stücks im Detail voneinander abweicht, ergeben sich bemerkenswerte Gemeinsamkeiten, und zwar der anfängliche Aufstieg $f-a-c^{\prime}$ im 1. Modus (Perfectio 1-2) sowie der Zielschritt g-a (Perfectio 4-5). Die meines Wissens neueste Übertragung von "Cest la ius en la roi pree/Pro patribus“" in Hans Tischler, The Earliest Motets (to circa 1270). A Complete Comparative Edition, New Haven/London 1982, Bd. 2, S. 1521 f. übersieht diese Möglichkeit einer alternativen Kombination der Verse a und A mit der ersten oder zweiten Phase der gregorianischen Tonfolge und beschränkt sich jedes Mal auf deren sechs Anfangstöne. So erreicht der Tenor insgesamt nur den ersten Ton der zweiten Phase, während die Fortsetzung der zweiten und die dritte Phase gar nicht eingesetzt werden. 
benützen, der Erwartung, welche an den Ursprungsort eines Refrains gestellt werden muss (nämlich den Refrain in seiner Refrainfunktion zu zeigen), am ehesten gerecht.

Beispiel 3:
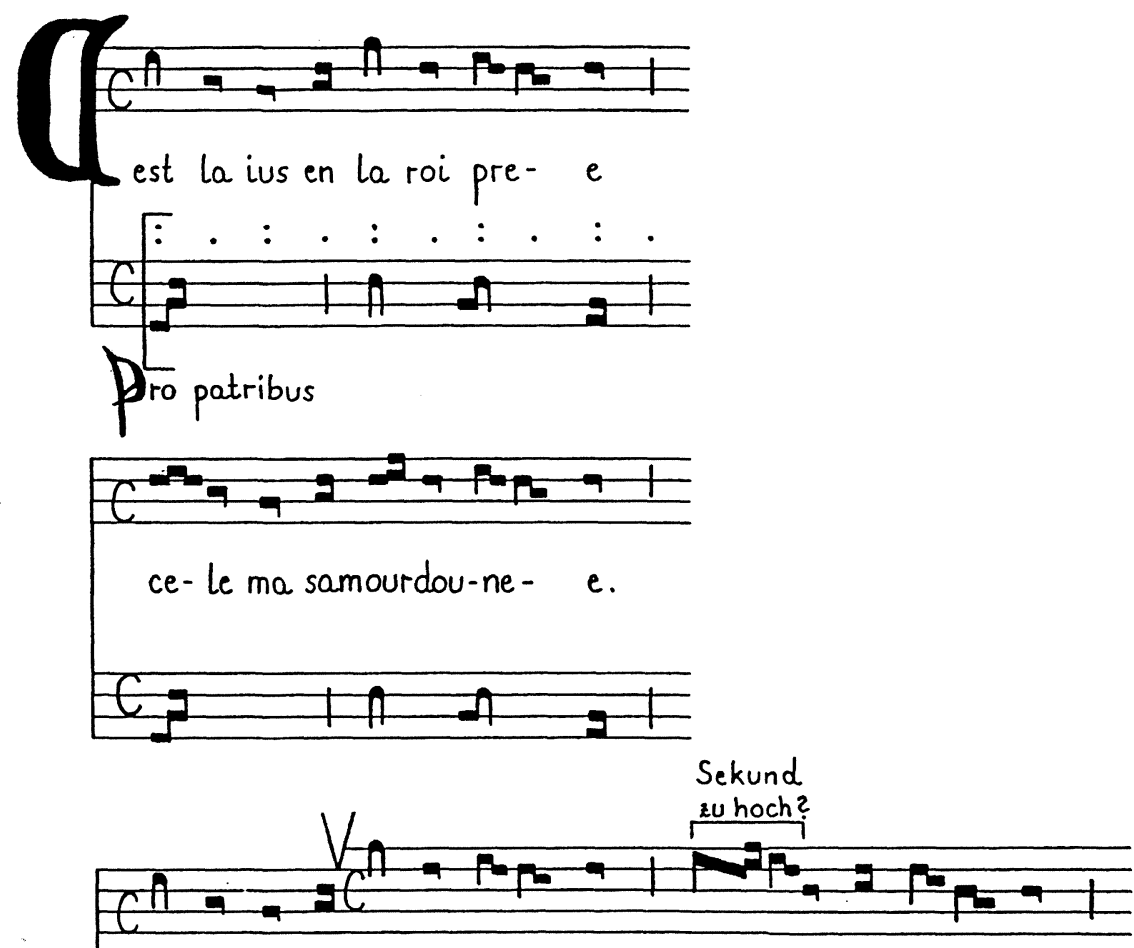

La fon-te-ne-L[e]i sort cle- re. fausvilainstraies en la.
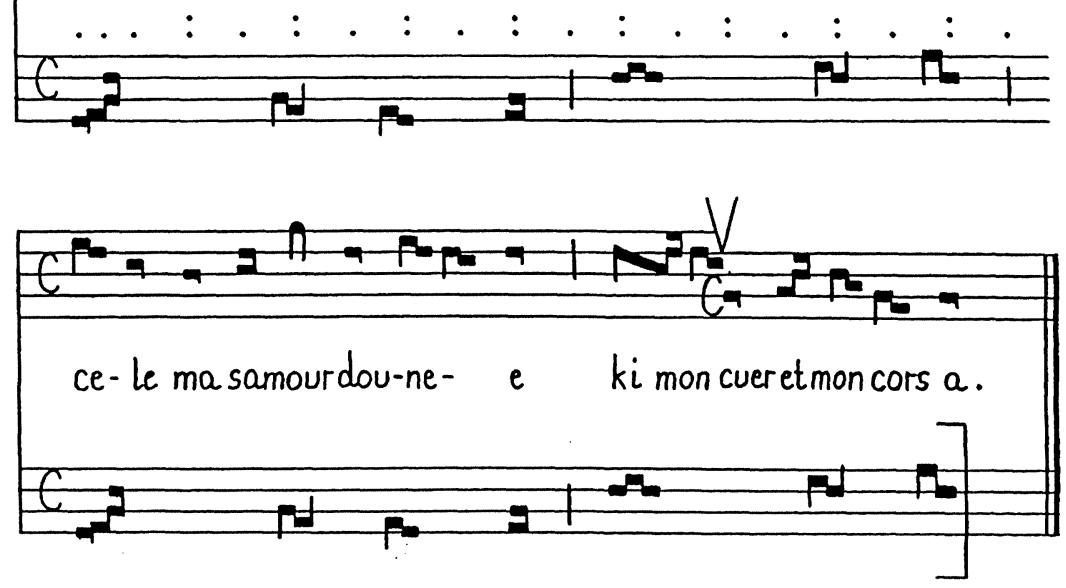

Nachschrift der Motette [403] aus der Handschrift N, f. $195^{\mathrm{r}}$ 
Es lässt sich also festhalten, dass die Wahrscheinlichkeit in der Tat dafür spricht, dass wir uns am Schluss der Notre-Dame-Klausel „Ne“ einem präexistenten Refrain gegenübersehen, der vom Urheber des Satzes als Zitat übernommen wurde. Restlos widerlegt ist Ludwigs These, dass der „,ele ma samor“-Refrain (ebenso wie alle anderen Refrains aus weltlichen Motetten, zu denen es eine Notre-Dame-Klausel gibt) erst durch nachträgliche Textierung der Klausel entstand, aber nicht. Doch vielleicht wäre die Beantwortung dieser Frage in einem tieferen Sinne gar nicht so erhellend, wie es in der derzeitigen Diskussion den Anschein hat. Vor allem dürfte relevant sein, dass die beiden Schlussordines, wie ausgeführt, ein typisches Refrainprofil zeigen. Dies bedeutet, dass wir im Bereich des Notre-Dame-Repertoires auf einen musikalischen Vorgang stoßen, der ungeachtet seiner Kombination mit dem Tenor gleichzeitig dem Vorbild aus der weltlichen Liedpraxis treu bleibt.

Dass hierbei Zusammenstöße oder wenigstens Reibungen von Tenor- und Duplumkonzept nicht auszuschließen sind (auch wenn es nur in Ausnahmefällen zu einer echten Niederlage des Tenors kommt, wie bei der Realisierung des vollständigen Rondeau in der Motette aus dem Chansonnier Noailles), lässt sich zu Beginn der Refrainphase erkennen, also dem $e^{\prime}-d^{\prime}-c^{\prime}-$ Abstieg des vorletzten Ordo. Um die Entsprechung mit dem $e^{\prime}-d^{\prime}$ - $c^{\prime}$-Abstieg des letzten Ordo auch im Tenor zu unterstützen, hat sich der Urheber des Satzes bemüht, das Melisma „Ne“ dergestalt zuzuordnen, dass es wiederum zu einer melodischen Entsprechung kommt: Beim ersten $e^{\prime}-d^{\prime}-c^{\prime}$-Abstieg des Duplum vollzieht der Tenor im Gegenzug einen Aufstieg über die Töne $g-a-c$ ', und dieselbe Wendung, lediglich um eine Quarte nach oben versetzt (also $c^{\prime}-d^{\prime}-f^{\prime}$ ), begleitet danach den zweiten $e^{\prime}-d^{\prime}-c^{\prime}$-Abstieg. Nun war jedoch für diesen Zusammenschluss des Tenors mit dem Duplum auch ein Preis zu zahlen: Die Refrainphase wird nämlich von einer imperfekten Konkordanz, der Sexte $g$-e', eröffnet, was einen musiktheoretisch gebildeten Musiker nicht vollends befriedigen konnte.

So haben wir es zu verstehen, wenn bereits die lateinische Motettenversion im selben Codex F, „Prothomartir plenus fonte“, ${ }^{15}$ wie auch die andere lateinische Motette zu unserer Klausel, ,Sederunt inique“ aus der Handschrift $\mathrm{W}_{2},{ }^{16}$ die Anfangstöne $e$ '- $d$ ' der Refrainphase zu $d^{\prime}$-e' umkehren, womit einerseits zwar eine ,saubere' Quintparallele zustande kommt, andererseits aber die für den Refraincharakter wichtige Entsprechung $e^{\prime}-d^{\prime}-c$ ' im vorletzten und letzten Duplumordo verloren geht. Dass erst die beiden Aufzeichnungen der französischen Motettenversion „En mai qant nest la rosee“ in den Quellen mit dem jüngsten Repertoirestand, $\mathrm{W}_{2}{ }^{17}$ und Mo, ${ }^{18}$ den Eingriff übernehmen, während nicht nur das Lied des Pierre de Corbie (im Chansonnier Noailles ebenso wie im Chansonnier du Roi), sondern auch alle anderen Motetten,

15. F, f. $410^{\mathrm{r}}-410^{\mathrm{v}}$

16. W, f. $185^{\mathrm{v}}$. - Wie F, so wurde auch der heute in der Herzog-August-Bibliothek Wolfenbüttel liegende Codex DW Guelf. 1099 Helmst. [W, ], unter dem Titel Wolfenbüttel 1099 Helmstadiensis-(1206) $W_{\text {, }}$, von Luther Dittmer innerhalb der Reihe Veröffentlichungen mittelalterlicher Musikhandschriften im Faksimile herausgegeben (Band 2; New York 1960).

17. $\mathrm{W}$, f. $226^{\mathrm{v}}$.

18. F-MOf, H196 [Mo], f. $247^{\mathrm{v}}-248^{\mathrm{r}}$. - Siehe das im ersten Band der Ausgabe von Yvonne Rokseth, Polyphonies du XIIle siècle. Le manuscrit H 196 de la Faculté de Médecine de Montpellier, Paris 1935, enthaltene Faksimile der Handschrift. 
die den Refrain enthalten, und sogar noch die früheste Aufzeichnung der französischen Motettenversion in der Handschrift MüA bzw. den ihr zugehörigen Wolf-Fragmenten ${ }^{19}$ die anscheinend ursprüngliche Form $e^{\prime}-d^{\prime}-c$ ' verwenden, darf als weiteres Indiz dafür gelten, dass wir es bei unserer Klausel mit einem der französischen Motette und ihren Schlussrefrains zumindest nahe stehenden Gebilde zu tun haben. Es bestätigt daneben aber aufs Neue, dass die Pflege und Überlieferung der französischen Motette dem Einfluss der geistlichen Musik und der mit ihr zusammenhängenden Musiktheorie ausgesetzt war, wobei niemals vergessen werden sollte, dass ohne geistliche Musik und Musiktheorie überhaupt eine zweistimmige Konstruktion wie unser „Ne“-Satz - egal, ob als Klausel oder Motette - nicht denkbar ist.

Das konstruktive Moment, welches in der Verknüpfung des zweimaligen $e^{\prime}-d^{\prime}-c^{\prime}$-Abstiegs im Duplum mit den melodisch gleichen Tenorwendungen $g-a-c$ ' und $c^{\prime}-d^{\prime}-f^{\prime}$ am Ende des „Ne“Satzes aufscheint, ist aber besonders für seinen Anfang von entscheidender Bedeutung. Hier entwickelt das Duplum, ausgehend von einer Umkehrung $e^{\prime}-f^{\prime}-g^{\prime}-e^{\prime}-c^{\prime}$ des Refrainbeginns, über dem je drei Perfectiones in einem Ordo zusammenfassenden Tenor eine symmetrische Anlage aus $5+4+4+5$ Perfectiones, die zweimal - in ihrer Mitte und an ihrem Ende - zu einer gemeinsamen Zäsur mit dem Tenor führt $(5+4$ bzw. $4+5=3+3+3)$. Die Geschlossenheit der symmetrischen Anlage wird durch eine Stimmtauschkonstruktion an den beiden Zäsurstellen bestätigt. So übernimmt das Duplum am Ende seines zweiten Ordo, d. h. vor der ersten Zäsur, den am Ende der symmetrischen Anlage stehenden Tenoraufstieg vom $g$ ins $c^{\prime}$, der uns ja aus der Refrainphase schon bekannt ist, während der Tenor gleichzeitig aufgrund des angewandten Rhythmusmodells die zugehörige Duplumbewegung in ihrer musiktheoretisch ,sauberen ' Form $d^{\prime}-e^{\prime}-c$ ' bringen kann, die wiederum am Ende des vierten Ordo, d. h. vor der zweiten Zäsur, vom Duplum aufgegriffen wird.

Im übrigen Verlauf der „Ne“-Klausel tritt das konstruktive Moment zurück, und es breitet sich ein längerer Mittelteil aus, in dem das Duplum in freier Folge vier und fünf Perfectiones umfassende Ordines aneinanderreiht. Die somit für das ganze Stück gültige Ausdehnung der melodischen Phrasen von vier bzw. fünf Perfectiones, deren Ausgangspunkt ohne Zweifel in der Refrainphase am Ende zu suchen ist, bietet jedoch, bei stets beibehaltenem 1. Modus, einem der häufigsten Verse der weltlichen Liedkunst wie der französischen Motette, nämlich dem

19. Die Bezeichnung MüA erhielt die Handschrift von Friedrich Ludwig, da ihm zur Zeit der Abfassung seines Repertorium, Bd. I/1, lediglich zwei in der Bayerischen Staatsbibliothek München unter der Signatur Cod. gall. 42 liegende Doppelblätter bekannt waren; dort befindet sich der Anfang unserer Motette auf der vierten Seite des zweiten Doppelblatts. Erst in seinem Aufsatz „Die Quellen der Motetten ältesten Stils“, in: AfMw 5 (1923), S. 187-190 konnte Ludwig auf die Existenz weiterer Fragmente aus MüA hinweisen, die damals im Besitz von Johannes Wolf waren, mit dessen Berliner Privatbibliothek sie jedoch im Zweiten Weltkrieg vernichtet wurden. Trotzdem haben sich die Wolf-Fragmente glücklicherweise in photographischen Reproduktionen der Pariser Bibliothèque nationale unter der Signatur Vma 1446 erhalten, die Luther Dittmer in seinem Aufsatz „The Lost Fragments of a Notre Dame Manuscript in Johannes Wolf's Library“", in: Aspects of Medieval and Renaissance Music. A Birthday Offering to Gustave Reese, hrsg. von Jan LaRue, New York 1966, S. 122-133 bzw. dem nach S. 430 eingebundenen Abbildungsteil des Bandes veröffentlicht hat; sie zeigen auch die Fortsetzung unserer Motette mit dem Motetusschluss und dem folgenden Tenor (siehe Plate 8, unten rechts, bei Dittmer). Die zwei Doppelblätter aus der Münchner Sammlung waren von Dittmer bereits Jahre vorher in Eine zentrale Quelle der Notre-Dame Musik (= Veröffentlichungen mittelalterlicher Musikhandschriften 3), New York 1959, im Faksimile mitgeteilt worden, das die „En mai“-Motette auf S. 27 jedoch nur in mangelhafter Qualität bietet; eine bessere Reproduktion legte inzwischen Rita Haub, Die Motetten in der Notre-Dame-Handschrift MïA (Bayer. Staatsbibl., Cod. gall. 42) (= Münchner Editionen zur Musikgeschichte 8), Tutzing 1986, S. 176 vor. 
männlichen oder weiblichen Siebensilber, Raum, so dass nicht nur am Anfang und Ende des Satzes, sondern auch in seinem Mittelteil der weltliche Pulsschlag spürbar bleibt. Außerdem lässt ebenso die Großanlage des Stücks aus fest gefügtem Eröffnungskomplex von insgesamt vier Duplumordines, einer lockeren Aneinanderreihung weiterer Phrasen in einem längeren Mittelteil und abschließender Refrainsituation deutlich ein dreiteiliges Konzept erkennen, das eine Vielzahl französischer ,Ars antiqua'-Motetten bestimmt, seine klarste Ausprägung aber in der ,Motet enté-Form findet, welche die zwei Hälften eines vorgegebenen Refrains auf Beginn und Ende des ansonsten frei entwickelten Motetus verteilt. Im französischen Text zu unserer „Ne“-Klausel ist diese Dreiteilung geradezu vorbildlich realisiert: Nachdem die vier Anfangsverse allgemein das Thema des Gedichts umrissen haben, beginnt der ,Ich“-Erzähler mit dem Ausruf „he diex“ im fünften Vers, von der eigenen Lage zu berichten und den Hörer an seinem Schicksal teilhaben zu lassen. Dieser Mittelteil mündet schließlich in den Refrain, der die persönliche Situation des ,Ich'-Erzählers gewissermaßen , auf den Punkt bringt' ${ }^{20}$

En mai quant neist la rousee que gelee sen reva.

garis est qui amie a.

car sa ioi en est doublee.

he diex mes cuers que fera.

coument tenir se porra.

tant est ma ioie doublee.

quant cele qui mon cuer a.

que lonc tens ai deservie

cele ma samor dounee

qui mon cuer et mon cors a.
Im Mai, wenn Tau die Blumen netzt und die Fröste weichen, ist froh, wer eine Freundin hat, denn zweifach ist sein Glück.

Oh Gott! Mein Herz, was wird es tun?

Wie kann es soviel Freude tragen?

Denn mein Glück ist übergroß:

Die mich ganz gewonnen hat, der ich lange schon gehöre, hat mir ihre Gunst geschenkt. Meine Seele und mein Körper sind ihr ewiges Eigentum.

Beispiel 4:

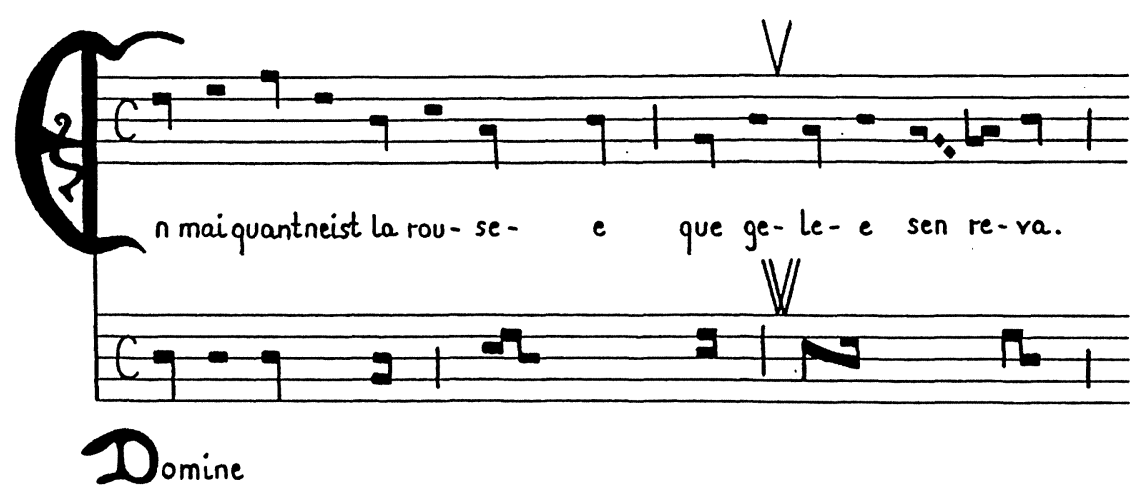

20. Die vom Autor dieses Beitrags verfasste Nachdichtung in deutscher Sprache ist dem Booklet zur CD Hare hare hye. Motetten des 13. Jahrhunderts des von ihm gegründeten Ensembles "Schola vocalis" (Nürnberg: MDL ErikaMusikverlag, 1992) entnommen. 


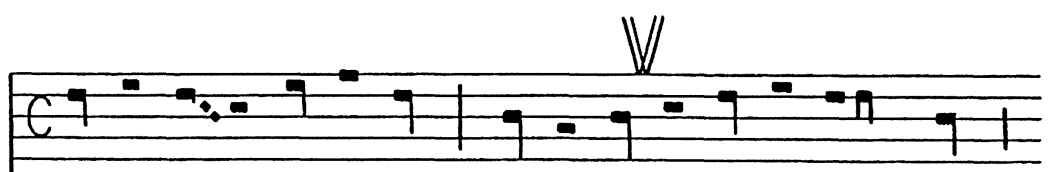

garis est qui a-mie a. car sa ioie en est double- e.
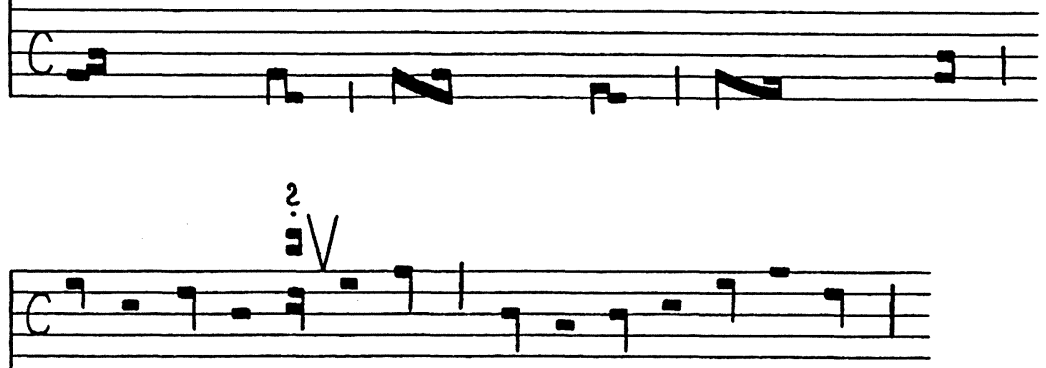

he diex mescuersquefe-ra. coument te-nir se por-ra.

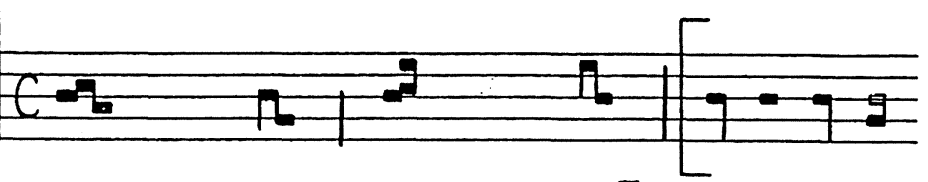

- Jterum.

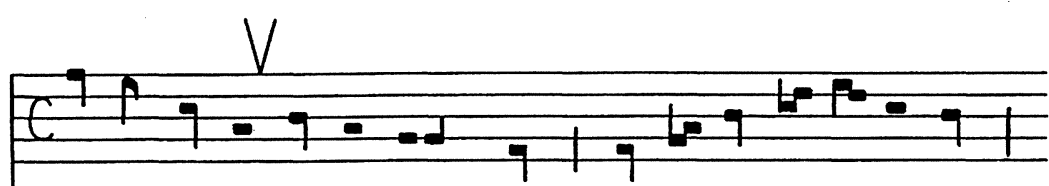

tant est ma ioi-e double- e. quant ce-le qui moncuer a.
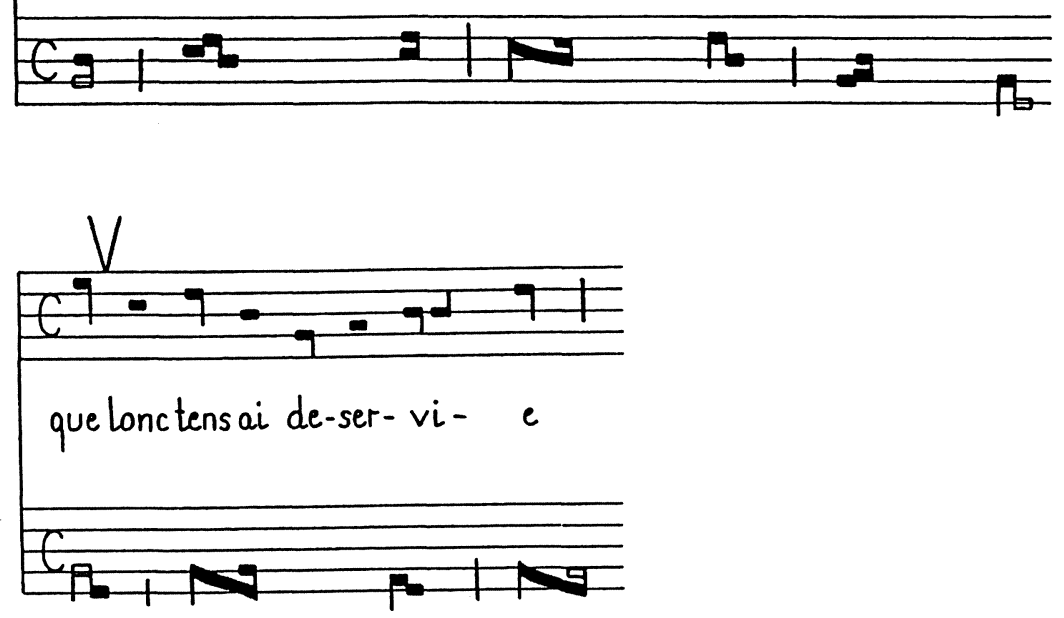


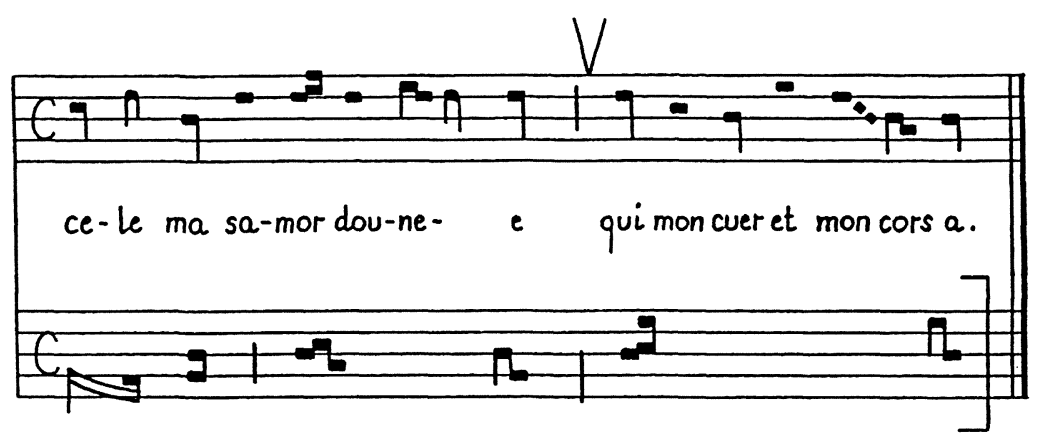

Nachschrift der Motette [62]

aus der Handschrift Mo, f. $247^{\mathrm{v}}$

Deutet also auch die musikalische Dreiteilung des „Ne“-Satzes auf die französische Motette „En mai qant nest la rosee“ als ursprüngliche Version, so könnte man weiterhin die Frage stellen, ob nicht dieses typische Formschema der französischen Motette seinerseits von der weltlichen Liedkunst angeregt ist. Immerhin steht am Anfang der ,Kanzonenstrophe ‘, der sich sehr viele Troubadour- und Trouvèrelieder des 12. und 13. Jahrhunderts bedienen, ein geschlossener Block aus vier Verszeilen, dem in loser Folge dann weitere Verse angefügt werden. Mündet jedoch die Strophe zuletzt in ein Refrainzitat, wie es bei den Liedern mit Wechselrefrains und damit auch in der Pastourelle von Pierre de Corbie geschieht, die am Schluss der ersten Strophe unseren „cele ma samor"-Refrain enthält, so liegt im Grunde ja dieselbe Disposition wie in der französischen Motette vor. Aber selbst die zahllosen Lieder, deren Strophenschluss auf einen Refrain verzichtet, kennen doch nichtsdestoweniger seine melodische Bezugnahme auf den Eröffnungsblock ${ }^{21}$ - ein formaler Kunstgriff, zu dem unser „Ne“-Satz, wie wir sehen konnten, in besonders konstruktiver Art greift, wenn das Duplum mit einer Umkehrung des Refrainbeginns $e^{\prime}-d^{\prime}-c^{\prime}-e^{\prime}-g$ ' anhebt.

Die im französischen Motettentext wie in der musikalischen Faktur nachweisbare Dreiteilung ist gewiss das stärkste Argument, das bei unserem Beispiel für eine Priorität der französischen Motette vor dem Klauselstadium und den lateinischen Motetten spricht. Darüber hinaus treten in der Notierung der Klausel einige Unregelmäßigkeiten auf, die am ehesten nachvollziehbar sind, wenn man die französische Motette als Vorlage annimmt. ${ }^{22}$ Halten sich die

21. Es sei daran erinnert, dass dieses von Gennrich, Grundriß einer Formenlehre des mittelalterlichen Liedes als Grundlage einer musikalischen Formenlehre des Liedes (Halle 1932), S. 245-249 als ,Rundkanzone' bezeichnete Modell der Troubadour- und Trouvèrekunst auch in den deutschen Minne- und Meistersang übernommen wurde. Ausgehend von den mittelalterlichen Vorbildern wirkte es auf die nachfolgende Produktion weltlicher Lieder und, offenbar über die Gattung des Tanzlieds, auf die formale Gestaltung von Tanzmelodien ein (daneben, speziell in Deutschland, auf den protestantischen Gemeindechoral). Besonders durch die Erweiterung einstimmiger Abläufe zu mehrstimmigen Sätzen im Tanzbereich lebte es dann bis in das 18. Jahrhundert fort. Damit konnte das alte Liedkonzept schließlich in der ,Sonatenhauptsatzform " noch die große Instrumentalmusik der Wiener Klassik und der Romantik befruchten.

22. Auf die Tatsache, dass Unregelmäßigkeiten beim Einsatz der rhythmischen Notenschrift, sine littera" im Fall unseres „Ne“-Satzes sowie 19 weiterer Notre-Dame-Klauseln für die Ableitung von einer Motette sprechen, machte - wie schon zu Beginn erwähnt - zuerst William G. Waite in The Rhythm of Twelfth-Century Polyphony, S. $100 \mathrm{f}$. aufmerksam. 
ersten drei Duplumordines streng an die Gepflogenheiten der modalen Notation (die im zweiten und dritten Ordo auftretenden Zusätze resultieren jeweils aus einer ,fractio modi ‘, wie von der Musiktheorie des 13. Jahrhunderts mehrfach beschrieben), so werden im vierten Ordo die beiden letzten Binariae aufgebrochen, indem die übliche Abfolge des 1. Modus aus Ternaria und nachfolgenden Binariae (hier mit Plica am Ende der vorletzten Binaria) zu 3331 geändert wird;

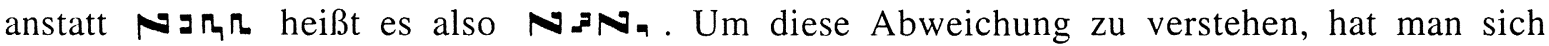
klarzumachen, dass der vierte Ordo zu den längeren Ordines mit fünf Perfectiones gehört, deren vorletzte Perfectio den Vortrag des weiblichen Siebensilbers in der französischen Motette auf eine Longa perfecta abbremst. Nachdem der erste, ebenfalls fünf Perfectiones einnehmende Ordo jedoch die vorletzte Perfectio in Klausel und Motette mit einem einzigen Ton füllte, setzt der vierte Ordo erstmals eine Verzierung ein, welche vom $e$ ' ausgeht und über $d$ ' ins $e$ ' zurückkehrt. Durch die Änderung der üblichen Ligaturenkonstellation wird dieses $e$ '- $d$ '- $e$ '-Ornament als eigenes Element sichtbar, dem auf der Textebene gleichfalls nur eine Silbe zugeordnet ist. Konsequenterweise wird auch in Ordo 7, dem nächsten Ordo mit fünf Perfectiones, die vorletzte Perfectio als eine separate Ligatur (diesmal mit zwei Tönen) notiert; in der Textüberlieferung aus Mo endet der zugehörige Vers sogar mit demselben Wort „doublee“ wie Ordo 4.

Natürlich stellt sich dann die Frage, warum der Notator nicht den ersten Refrainordo, wie den vierten Ordo, ebenso mit drei Ternariae und abschließender Simplex geschrieben hat. War es ihm wichtiger, das präexistente Versatzstück aus der weltlichen Musikpraxis mit den Mitteln seiner rhythmischen Notation möglichst genau zu erfassen, und fühlte er sich etwa zu dieser Vorgehensweise umso mehr legitimiert, als dadurch der bis zum Hochton $g$ ' vordringende Schlüsselbegriff des gesamten Textes, ,samor", in einer Binaria vereinigt wurde? Dass ihm der französische Motettentext wenigstens, in einem Augenwinkel ' gegenwärtig war, scheint jedenfalls die dritte Auffälligkeit der F-Notierung zu bestätigen. So spaltet er die eigentlich korrekte Ternaria zu Beginn des fünften Ordo auf und nähert die Tonschritte $f^{\prime}-d^{\prime}$ und $e^{\prime}-c^{\prime}$ optisch einander an, indem er zwei Clives schreibt, an deren zweite noch ein Quadrat mit aufsteigendem Plicastrich angesetzt ist, obwohl nicht nur eine reguläre Schreibung des 1. Modus aus Ternaria und Binaria mit aufsteigendem Plicastrich möglich gewesen wäre, sondern auch die der anfänglichen Clivis folgende Gruppe $e^{\prime}-c^{\prime}-d^{\prime}-e$ ' nach dem üblichen Usus der Notre-Dame-Handschriften als Porrectus mit auf die rechte Seite gewendetem $d^{\prime}$-Quadrat zur Anbringung des Plicastrichs erscheinen

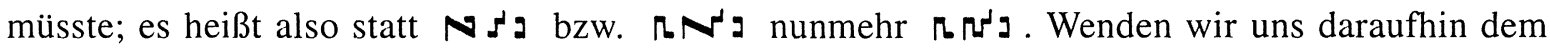
französischen Motettentext zu, bemerken wir, dass analog zu den beiden Clives auch hier vom Dichter zwei Sinneinheiten nebeneinander gestellt sind: „he diex“ und „mes cuers“. Freilich könnte man dagegenhalten, dass die Schreibung in Clives nicht notwendigerweise durch den Text ausgelöst wurde, da bereits der melodische Ablauf an dieser Stelle zwei gleich gerichtete Terzintervalle, $f^{\prime}-d$ ' sowie $e^{\prime}-c$ ', zeigt. Abgesehen davon, dass eine solche Konstellation jedoch kaum genügt hätte, um die Normalität der modalen Schrift zu verlassen, deutet der enge Zusammenhang von Melodie- und Textstruktur selbst abermals in Richtung des Motettenprimats.

Nun beschränkt sich der Zusammenhang von Melodie- und Textstruktur, wie wir gesehen haben, keineswegs auf derartige Details, sondern wirkt sich zuerst in der Übereinstimmung der 
Ordolängen von vier und fünf Perfectiones mit den männlich und weiblich endenden Siebensilbern, aber auch der Koordination von musikalischer Formanlage und inhaltlicher Gliederung aus. Weil jedoch der kundige Hörer bei der Aneinanderreihung melodischer Phrasen von vier und fünf Perfectiones in einem dreiteiligen Konzept aus fest gefügtem Eröffnungsblock, fließender Fortsetzung und refrainmäßig gebautem Schlussglied kaum anders kann, als den Vortrag weltlicher, dem Trouvèreschaffen verwandter Dichtung in französischer Sprache mitzudenken, tragen diese Merkmale dazu bei, dass nicht allein der Motetus „En mai qant nest la rosee“, sondern auch seine textlose Gestalt als Duplum der „Ne“-Klausel den Eindruck einer ihrem eigenen Gesetz verpflichteten und damit in sich schlüssigen Liedmelodie vermittelt. ${ }^{23}$ Ungeachtet der konstruktiven Verknüpfung mit dem Tenor ist der Duplum- bzw. Motetusablauf sogar außerhalb des zweistimmigen Satzes vorstellbar, und er verdankt diese potentielle Selbständigkeit der tonalen Disposition.

So bewegt sich die gesamte Melodie in einem $c^{\prime}$-Raum, dessen Haupttöne - in erster Linie das c', seine Oberterz $e$ ' und seine Oberquint $g$ ' sowie die Unterquart $g$ - derart stark hervortreten, dass von einer stabilen Klangsituation gesprochen werden kann, die durch das melodische Geschehen selbst zustande kommt. Indem die Melodie jedoch fortwährend auf ihren eigenen Grundton c' bezogen bleibt, ist sie auf den Tenor als intervallischen oder klanglichen Bezugspunkt nicht angewiesen. Im Gegenteil: Es gelingt dem Tenor lediglich streckenweise, die ihm fremde c'-Tonalität des Duplum bzw. Motetus zu unterstützen, dort nämlich, wo er seinerseits um die $c^{\prime}$-Station als Repercussa des fünften Kirchentons kreist, während er mit dem Absteigen zur Finalis $f$ ein anderes Gewicht setzt, das sich jenseits des Horizonts von Duplum bzw. Motetus befindet. Es hat den Anschein, als würden in unserem Satz mit dem $f$-Raum des gregorianischen Chorals und dem eine Quint höher liegenden c'-Raum der Zusatzstimme zwei Welten aufeinanderstoßen, und nachdem in einem einfachen Klangraum mit Grundton, Oberterz und Oberquint ruhende Melodien im französischen Lied des 13. Jahrhunderts in großer Anzahl begegnen, darf auch die klangliche Beschaffenheit des Duplum- bzw. Motetusablaufs schließlich als Einfluss musikalischer Vorstellungen aus dem weltlichen Bereich und damit als Indiz für die Priorität der französischen Motette aufgefasst werden.

Durch die gründliche Beschäftigung mit dem „Ne“-Satz in seiner Version als Klausel und französische Motette ist es also möglich, den Verdacht von Frobenius, dass die Klausel auf der französischen Motette beruht, zu erhärten. ${ }^{24}$ Gleichzeitig treten jedoch wesentliche Eigenschaften

23. Die Affinität gewisser Notre-Dame-Klauseln zum weltlichen Lied wurde auch von Page, The Owl and the Nightingale, S. 152 bemerkt (,It is only necessary to sing these pieces today to feel the presence of the latent secular songs which musicians in the early thirteenth century seem to have sensed for themselves“), ohne dass daran jedoch Überlegungen zum „genetischen Verhältnis" von Klausel und französischer Motette geknüpft werden.

24. Im Unterschied dazu vertritt Mark Everist, French Motets in the Thirteenth Century, S. 101-104, ebenso wie bereits in "The Rondeau Motet: Paris and Artois in the Thirteenth Century", S. 16, noch sieben Jahre nach Frobenius die Auffassung, dass "The clausula from Florence serves as a model for the two-part French motet (62) En mai que neistDomine (M3)“, und hält es damit auch für ,relatively certain“, dass „The music of the refrain used in the rondeau-motet (403) C'est la jus en la roi-Pro patribus (M30) originated in a Notre-Dame clausula“, obgleich diese Motette, wie ich ausgeführt habe, „von allen Stücken, die den Refrain benützen, der Erwartung, welche an den Ursprungsort eines Refrains gestellt werden muss (nämlich den Refrain in seiner Refrainfunktion zu zeigen), am ehesten gerecht" wird. 
von Musik und Text ins Bewusstsein, die über Refrainzitat und Dehnung des Tenorrhythmus am Ende weit hinausgehen. ${ }^{25}$ Obschon es sich im vorliegenden Fall, da eine französische Motette als ursprüngliche Fassung anzunehmen ist, wohl mit Sicherheit um ein musikalisch fertig in das Notre-Dame-Repertoire importiertes Stück handelt, belegt diese Aneignung nichtsdestoweniger die Offenheit der damaligen Musiker an der Kathedrale von Paris für musikalische Vorstellungen aus dem weltlichen Bereich. Deshalb ist es mehr als unwahrscheinlich, dass sich die Rolle der von außerhalb des Kirchenraums übernommenen Vorbilder auf fertige Stücke beschränkt hat. Wir müssen uns fragen, zu welchem Zeitpunkt jeweils das Aussehen der Notre-Dame-Musik durch eine weltliche Orientierung verändert wurde, und wie solche Veränderungen vor sich gingen.

Unter diesem Aspekt wären jedoch die das Schaffen der Notre-Dame-Schule enthaltenden Quellen nochmals einer umfassenden Betrachtung zu unterziehen, um ein möglichst klares Bild von den verschiedenen Entwicklungsstufen der Notre-Dame-Musik zu gewinnen. Das somit entstehende Grundgerüst hätte dann als Voraussetzung für das Herstellen der Bezüge ,nach außen', d. h. in den weltlichen Bereich, zu dienen. Dabei müsste nicht allein versucht werden, die Grenze zwischen der Aufnahme von fertigen Stücken und dem schöpferischen Umgang mit den Anregungen aus der französischen Lied- und Motettenkunst festzulegen, auch verschiedene Phänomene, die im Rahmen der Notre-Dame-Musik auffallen, könnten in neuem Licht erscheinen. Hier ist natürlich an die rhythmischen ,Modi ‘ zu denken, die uns einerseits von der zeitgenössischen Musiktheorie als ,gelehrtes System" präsentiert werden, obwohl ihre Entstehung auf das scholastische Denken kaum angewiesen war. Man sollte aber auch das klangliche Pendeln, das sich wiederholt in den Organalpartien der drei- und vierstimmigen Notre-Dame-Organa ansiedelt, auf sein Verhältnis zum gregorianischen Choral hin überprüfen: Jedenfalls nutzt es den regungslosen Halteton des Tenors, um völlig andere, eigenständige Musik anzubringen (womit es dem autonomen Gepräge des „En mai“-Motetus ähnelt), und da den beteiligten Sängern aufgrund der regulären Zeitabsteckung des Pendelvorgangs möglich ist, auch ohne notenschriftliche Vorausplanung am musikalischen Geschehen teilzunehmen, verweist das klangliche Pendeln erneut auf den außerkirchlichen Bereich als Ursprungsort. Diese Sphäre des weltlichen Musizierens scheint im berühmten „Sommerkanon“ aus England aber tatsächlich greifbar zu werden, wo der Pendelvorgang - gemeinsam mit den melodischen Elementen, die er auslöst - in verwandter Form begegnet. ${ }^{26}$

Zweifellos wäre es angebracht, in eine Untersuchung der weltlichen Einflüsse auf die Notre-Dame-Musik auch deren eigene Liedgattungen einzubeziehen, und hier neben dem einund mehrstimmigen Conductus namentlich die rondeaumäßig gebauten Lieder im

25. Derartige Beobachtungen an der Notre-Dame-Musik selbst verleihen den Ausführungen von Page, The Owl and the Nightingale, S. 144-154 über die „magistri organici“ besonderes Gewicht, denn sollten Leoninus und Perotinus diesem Musikerstand angehört haben, wie Page behauptet (ebd., S. 151), so müsste man tatsächlich davon ausgehen, dass sie Kontakte zur weltlichen Musikpraxis unterhielten.

26. Ausführlich werden die mit dem klanglichen Pendeln in den drei- und vierstimmigen Notre-Dame-Organa und dem „Sommerkanon“ einhergehenden Fragen im Rahmen meiner Dissertation Klang und Konstruktion in der englischen Mehrstimmigkeit des 13. Jahrhunderts. Ein Beitrag zur Erforschung der Stimmtauschkompositionen in den WorcesterFragmenten (= Münchner Veröffentlichungen zur Musikgeschichte 47), Tutzing 1990, erörtert. 
Schlussfaszikel von F. Wie das Beispiel „Pater creator omnium“27 deutlich macht, müssen wir dort auf melodische Strukturen gefasst sein, deren Ähnlichkeit mit dem liedhaften Profil des „En mai“-Motetus geradezu ins Auge sticht. Besonders der Refrain „Deus in adiutorium/nostrum venit intendere“ am Ende füllt den Quintraum über $g$ in derselben Weise wie der Anfangsvers des „,cele ma samor"-Refrains. Dass die Refrainmelodie des lateinischen Liedes dann aber mit einer Duplumstelle des dreistimmigen „Benedicamus domino“-Organum Nr. $6^{28}$ fast identisch ist, liefert den Beweis, dass auch innerhalb des Notre-Dame-Repertoires durchaus Verbindungen zwischen eingängigem Lied und reifer Organalkunst bestehen. Dieser Kontakt wird eindrucksvoll durch ein anderes Refrainlied des Schlussfaszikels von F unterstrichen: das dem „Pater creator omnium“-Beispiel vorausgehende „Qui pro nobis mori non respuit“, denn bei ihm haben wir es lediglich mit einer Textierung des Duplum aus dem zweiten „Alleluya“ des Organum „Alleluya. Dies sanctificatus“29 zu tun, dessen Schlussteil wiederholt wird, um die Rondeauform zu vervollständigen. Dass aber die Liedversion als Bearbeitung des melodischen Vorgangs im Organum anzusehen ist, zeigen nicht nur die sperrige Deklamation und das beibehaltene Wort „,alleluya“, sondern auch der Umstand, dass die Verlagerung des tubamäßigen Hochtons c' nach a zu Beginn der zweiten Liedhälfte tonal unbestimmt bleibt, wenn der gleichzeitige $c$ - $d$-Schritt des Tenors fehlt.

So lässt uns das Refrainlied „Qui pro nobis mori non respuit“ im Schlussfaszikel von F noch einmal an den Ursprung der Motette zurückdenken: die vorerst lateinische Tropierung von rhythmischen Partien der Notre-Dame-Organa, denen bald jedoch französische Texte folgen sollten, die mit einer Herauslösung der nun Text tragenden Abläufe aus dem ursprünglichen Kontext und ihrer Verselbständigung zu einer eigenen Kompositionsgattung der weltlichen Musik verbunden sind. Damit trat die Pflege der lateinischen Motette für den Zeitraum von mehreren Jahrzehnten in den Hintergrund, bis konservative, einer, verweltlichten“ Motette feindlich gesinnte Kirchenkreise um 1300 ihre Wiederbelebung betrieben und die Gattung so dauerhaft ins geistliche Ambiente zurückholten. Indem lateinische und französische Motette dergestalt auf unversöhnliche Weise gegeneinander stehen, kann die Situation der Gattung an der Wende vom 13. zum 14. Jahrhundert als ein Reflex auf die philosophische Diskussion um die Schriften des Aristoteles verstanden werden, die an der Universität von Paris namentlich in den 1260er Jahren vehement geführt wurde und mit der Polarisierung in einen ,rasanten Säkularismus“ und die ,prinzipielle Unweltlichkeit der Konservativen“30 zu erbittertem Streit ausartete.

Ebenso wie der damals aus Italien zu Hilfe gerufene Thomas von Aquin seit 1268 um eine Versöhnung der feindlichen Positionen bemüht war und einen, mittleren Weg' einschlug, gelang es aber einem speziellen Motettentypus des ausgehenden 13. Jahrhunderts, die alte Einheit von

27. F, f. $467^{r}$.

28. F, f. $42^{r}$, drittes System (auf ,-mus“).

29. F, f. $101^{\mathrm{r}}$, viertes und fünftes System.

30. Josef Pieper, Scholastik. Gestalten und Probleme der mittelalterlichen Philosophie, München ${ }^{2} 1981$, S. 111. 
Geistlichem und Weltlichem zurückzugewinnen: Gemeint ist die zweisprachige Doppelmotette, die in simultanem Vortrag eine lateinische und eine französische Dichtung miteinander verbindet, wobei die Stimmen oftmals auch musikalisch differenziert werden. Mit diesem Bekenntnis zur unauflösbaren Verbindung von, Gott und Welt" veranschaulicht sie gewissermaßen die Ideen des Scholastikers, dem zufolge die Bejahung dessen, was durch den Verstand zu erkennen und mit den Sinnen zu erfühlen ist, nicht als Abwendung von der göttlichen Wahrheit verurteilt werden darf, wie umgekehrt „die bejahende Zuwendung zur natürlichen Weltwirklichkeit und zur natürlichen Wirklichkeit im Menschen selbst letztlich nicht anders als theologisch begründet und gerechtfertigt werden kann".31

Demgegenüber war das frühe 13. Jahrhundert auf die Vermittlung eines Thomas von Aquin noch nicht angewiesen, um die Einheit von Geistlichem und Weltlichem zu realisieren. So war es offenbar auch für Musiker und Dichter selbstverständlich, dass die beiden Sphären in fruchtbarer Symbiose zusammenlebten. Davon legt die Entstehung der französischen Motette aus dem Klauselsatz der Notre-Dame-Schule beredtes Zeugnis ab, und ebenso standen die NotreDame-Musiker selbst der weltlichen Musik anscheinend offen genug gegenüber, um sich von ihren Ausdrucksformen in mannigfacher Weise anregen zu lassen. ${ }^{32}$ Wenn sie - wir sahen es am Beispiel unserer „Ne“-Klausel aus dem Codex F - die weltliche Motette ihrerseits in den Kirchenraum zurückholten, ist es deshalb kaum zu erwarten, dass auch sie damit eine restriktive Intention verbanden. Entscheidend dürfte vielmehr gewesen sein, dass die Aneignung weltlicher Vorbilder dazu angetan war, die eigene Musik um neue Facetten zu bereichern und sie auf solche Weise interessant und abwechslungsreich zu gestalten. Die damit einhergehenden Veränderungen aufzuspüren und zu beschreiben, ist jedoch ein faszinierendes Unternehmen, und es wird ohne Zweifel zu den Aufgaben einer künftigen Mediävistik im Fach Musikwissenschaft gehören, die Notre-Dame-Musik unter diesem Gesichtspunkt zu betrachten.

31. Pieper, Thomas von Aquin. Leben und Werk, München 1981, S. 122 f.

32. Bei dem von Page, The Owl and the Nightingale, S. 152-154 geäußerten Verdacht, dass die „magistri organici“, denen er Leoninus und Perotinus zurechnet (siehe Anm. 25), an der Entstehung der französischen Motette unmittelbar beteiligt waren, handelt es sich vorerst nur um eine - wenn auch besonders reizvolle - Hypothese, die noch zu verifizieren ist. Sollte sie zutreffen, würde die „Einheit von Geistlichem und Weltlichem“, die im Zusammenhang von Klauseln und lateinischen Motetten einerseits sowie französischen Motetten andererseits sichtbar wird, eine konkrete Begründung erfahren. 